\title{
The Gutenberg Algorithm: Evolutionary Bayesian Magnitude Estimates for Earthquake Early Warning with a Filter Bank
}

\author{
by M.-A. Meier, T. Heaton, and J. Clinton
}

\begin{abstract}
Earthquake early warning (EEW) is a race against time. In particular, at proximal sites to the epicenter (typically the most heavily affected sites), strong ground motion starts shortly after the $P$-wave onset. For these sites, regional-type EEW systems that wait until data from several stations are available before issuing a warning and that require fixed data windows following a trigger are not fast enough. Single-station algorithms, on the other hand, have high uncertainties that compromise their usefulness. In this article, we propose that uncertainties of the earliest warning messages can be reduced substantially if the broadband frequency information of seismic signals is fully exploited. We present a novel probabilistic algorithm for estimating EEW magnitudes. The Gutenberg algorithm uses a filter bank for a time-frequency analysis of the realtime signals and estimates the posterior probabilities of both magnitude and sourcestation distance directly from the observed frequency content. It starts off as a singlestation algorithm and then naturally evolves into a regional-type algorithm, as more data become available. Using an extensive near-source waveform data set, we demonstrate that the Gutenberg parameter estimates reach the estimation accuracy and precision of existing regional-type EEW systems with only $3 \mathrm{~s}$ of data from a single station. The magnitude estimates, however, saturate at a threshold magnitude that depends on the available signal length that is used for the estimation, suggesting that current EEW magnitude estimates (1) are observational rather than predictive and (2) have to be considered minimum estimates, depending on the amount of available data.
\end{abstract}

\section{Introduction}

Modern seismic networks with real-time data transmission streams allow the recognition of ongoing seismic events as soon as the seismic $P$ phases arrive at the nearest stations to the epicenter. Earthquake early warning (EEW) systems use the frequency content and absolute amplitudes of such waveforms to estimate event magnitudes and locations in near real time to warn more distant sites of imminent strong ground motions (Heaton, 1985; Allen and Kanamori, 2003).

Over the past decade, several damaging earthquakes have occurred in areas with operational EEW systems, revealing both the great potential as well as the shortcomings of current systems. For areas where seismic hazard predominantly comes from shallow crustal seismicity, one particular insight from these real-world examples is that, in most cases, the earliest warning messages are the most important ones. By the time essential updates are available, strong shaking has already begun at sites that will experience high groundmotion intensities as the event unfolds. This is because events in the magnitude range $M<7$ can produce severe but localized damage - the high intensity ground motions are confined to relatively small zones around the epicenter. At the strongly affected proximal sites, the high-amplitude ground motions from $S$ waves arrive soon after the $P$ waves that are used to detect and characterize the event; consequently, there is only a short time window for computing and issuing timely warnings.

Only in the rare cases of very large events, in which there are large areas that are far away from the epicenter but close to the finite rupture zone, will there be long warning times, allowing full use of the estimation updates. On the other hand, for the more frequent $M<7$ events, regional EEW approaches, which use a fixed number of stations (typically 4 or 6) and fixed time windows (typically 3-4 s) (e.g., Cua and Heaton, 2007; Satriano et al., 2011; Kuyuk et al., 2013) may be too slow in many scenarios. Kuyuk and Allen (2013) analyzed the blind zone of a hypothetical system for California that requires $4 \mathrm{~s}$ of data from at least one station and a $P$-wave detection at four stations to trigger. Including telemetry and processing delays of $4 \mathrm{~s}$, the resulting blind zone radii were 10-20 km in metropolitan San Francisco, 20-30 km in metropolitan Los Angeles, and $>30 \mathrm{~km}$ in less densely populated areas, depending mainly on network station density. However, according to the intensity prediction equations of Allen et al. (2012), it takes an $M>6.5$ earth- 
quake to produce strong intensities (VI on the modified Mercalli intensity scale) beyond a rupture distance of $30 \mathrm{~km}$. This shows that, in most cases, sites with intensities greater than or equal to VI would only get a timely warning in the case of an $M>6.5$ event. Using a similar system in Italy, Picozzi et al. (2015) conclude that positive warning times at sites with intensity ground motions larger than VII on the European Macroseismic Scale are only possible for events with $M_{\mathrm{w}}>6.5$.

Onsite-type approaches (e.g., Wu et al., 2007; Böse, Hauksson, Solanki, Kanamori, and Heaton, 2009; Böse, Hauksson, Solanki, Kanamori, Wu, and Heaton, 2009), on the other hand, can provide faster warning information, but they may not be accurate enough for many EEW applications. An optimal EEW algorithm, however, would not belong to either of these two categories. Instead, it would start characterizing an event as soon as a first station triggers and then assimilate all incoming information in a systematic wayand thus naturally evolve into a regional system (Kanamori, 2005). The uncertainties of the earliest earthquake characterizations when little data are available are likely very high but accurately represent the state of knowledge at a given instant.

A primary objective of an EEW algorithm, therefore, should be to attain the lowest possible uncertainty levels as soon as possible following the event detection. The usefulness of the system is then largely dependent on the level of these early uncertainties. In this study, we suggest that these early parameter uncertainties can be lowered substantially with respect to existing algorithms, if the available waveform information is more thoroughly exploited (Simons et al., 2006). We introduce a new magnitude estimation algorithm that is tailored to provide optimal estimates for the earliest of warning messages when only small amounts of data are available. The Gutenberg algorithm (GBA) performs a real-time time-frequency analysis of available waveforms via a minimum-phase-delay filter bank and, for each triggered station, estimates Bayesian posterior probabilities for magnitude and source-station distance combinations in an empirical way. Its probabilistic formulation offers a natural way to assimilate newly available data (from the same or from newly triggered stations), as well as external parameter constraints such as distance constraints from real-time epicenter locations or prior information. Furthermore, the algorithm quantifies the uncertainties of magnitude and source-station distance (including their interdependence) in a systematic way.

We first present an extensive near-source waveform data set that we have compiled to train and test the algorithm. We then explain the real-time time-frequency analysis via the filter bank and show how the algorithm uses the real-time frequency information for the magnitude estimation. This is followed by an analysis of the resulting time-dependent magnitude estimates and their uncertainties. We then discuss what the algorithm performance implies for the possibilities and the limitations of EEW at proximal sites.

\section{Waveform Data Set for Algorithm Training and Testing}

Our parameter inference method is empirical. We therefore have compiled an extensive waveform data set that we use as training and test data. The goals were to best quantify the dependency of the evolving peak ground-motion amplitudes on the EEW-relevant parameters magnitude $M$ and hypocentral distance $R$. For an EEW algorithm, the focus is to collate the maximum available information for near-source ground motions of large events. Because we also want to effectively distinguish these ground motions from other events, we include data from smaller events and from more distant recordings. Furthermore, the algorithm is designed for shallow crustal seismicity, as is predominant in California, Turkey, or Switzerland; we therefore only include shallow seismicity data. We strove to use maximally objective data selection criteria.

Japan

From the strong-motion networks K-Net and KiK-net, we use all records of events with $M_{\mathrm{JMA}} \geq 5.0$ and with hypocentral distances $<100 \mathrm{~km}$. To exclude subduction zone events, we excluded events with a focal depth $>25 \mathrm{~km}$ and all records of events for which the nearest record has a hypocentral distance of $>40 \mathrm{~km}$. Only records from surface stations were used. The resulting number of three-component records is 8095. Japan Meteorological Agency (JMA) magnitudes are used.

\section{Southern California}

From the Southern California Seismic Network data (SCSN) archive, we collect all high-rate broadband recordings (seed channel names HH[ENZ]) and high-rate strongmotion recordings (seed channel names H[NLG][ENZ]) with hypocentral distances $<100 \mathrm{~km}$ of events with catalog magnitudes $M \geq 4$ from the time period 1 January 1990-8 August 2014 and $M \geq 3$ from the time period 1 January 2001-18 August 2014. Furthermore, we include all records with hypocentral distances $<50 \mathrm{~km}$ and $M \geq 2$ from the time period 1 January 2012-18 August 2014. A total number of 55,454 three-component records was processed from southern California. In addition to the waveforms, we have also downloaded phase picks where available. We use the preferred magnitudes from the SCSN catalog, which are $M_{\mathrm{L}}$ and, for larger events, $M_{\mathrm{w}}$.

\section{Next Generation Attenuation-West 1}

From the Next Generation Attenuation-West 1 (NGAWest 1) strong-motion data set (Chiou and Youngs, 2008), we use all traces we are certain include the $P$-wave onset (911 out of a total of 3551 three-component recordings). For all of the used records we use $M_{\mathrm{w}}$ magnitudes. 


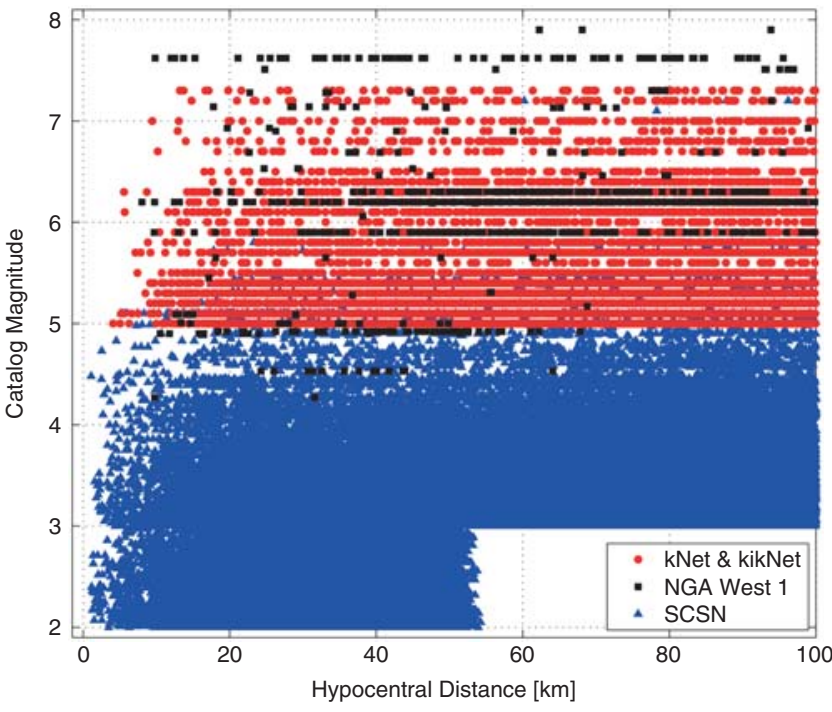

Figure 1. Catalog magnitudes and hypocentral distances of the 64,460 three-component records from a total of 5852 events.

We merge all these records into a common data format and preprocess them with a causal fourth-order Butterworth high-pass filter with a corner frequency of $0.075 \mathrm{~Hz}$. We integrate accelerograms to velocity and then run all traces through a short-term average/long-term average $P$-phase picker (e.g., Baer and Kradolfer, 1987). We subsequently optimize the obtained picks with the SBPx algorithm (see Data and Resources), which maximizes a ratio of integrated weighted amplitudes before and after a number of candidate picks. We perform several automated quality checks to assure high data quality. In particular, we discard the following records: (1) records with signal-to-noise ratios $<20 \mathrm{~dB}$, (2) records without all three components, (3) records with peak ground velocity (PGV) that deviate by $>3 \sigma$ from the ground-motion prediction equations (GMPEs) of Cua (2005), (4) records with sampling rates $<100$ samples/s, and (5) records that might be clipped based on the maximum reported counts. In this study, we have treated the waveforms from all regions equally. The resulting waveform data set consists of 64,460 records, with three-component traces each, from a total of 5852 events (Fig. 1). A summary of the data set is given in Table 1.

\section{Filter Bank}

We then pass all $193,380(=3 \times 64,460)$ traces of the data set through a filter bank. The filter bank consists of nine separate one-octave-wide causal fourth-order Butterworth band-pass filters with corner frequencies between 0.09375 and $48 \mathrm{~Hz}$ (Fig. 2a). The filters are applied in the time domain and can be run in real time on a sample-by-sample basis. The output $y_{k}$ of a Butterworth filter $h\left(a_{n}, b_{n}\right)$ with input waveform $x_{k}$ is a linear combination of the $N+1$ previous input and the $N$ previous output samples:

$$
y_{k}=\sum_{n=0}^{N} a_{n} x_{k-n}-\sum_{n=1}^{N} b_{n} y_{k-n},
$$

in which $k$ is the sample index; $a_{n}$ and $b_{n}$ are the filter coefficients; and $N$ is the filter order, which is $N=4$ in this case.

Because Butterworth filters are minimum-phase-delay filters, they are optimal for EEW purposes. Low frequencies are delayed more than high frequencies (Fig. 2b), but this relative delay corresponds to the minimum that is possible in any causal operation. This way the information from each frequency band becomes available at the earliest possible point in time.

For each velocity waveform trace of the data set, we obtain nine band-pass-filtered seismograms, on each of which we measure peak absolute amplitudes as a function of time since the $P$-wave onset. We term these maximum absolute amplitudes "narrowband peak ground velocities," PGV as $\operatorname{PGV}_{j}^{n b}(t)$, in which subscript $j$ denotes the filter band $(j=1, \ldots, 9)$. For each trace, we update the $\operatorname{PGV}_{j}^{n b}(t)$ in $0.5 \mathrm{~s}$ intervals following the trigger and save two $\operatorname{PGV}_{j}^{n b}(t)$ values per station per time step - one for the combined horizontal components (arithmetic mean) and one for the vertical component. The respective horizontal peak values for each individual component need not occur on the same sample $k$.

\section{Real-Time Parameter Inference}

Based on a set of real-time observations $\operatorname{PGV}_{j}^{n b}(t)$ from some individual target waveform, we then want to jointly estimate the most probable parameter values for magnitude $M$ and source-station distance $R$. We do this with a memory-

Table 1

Number of Three Component Records from Japanese Strong-Motion Data (K-Net and KiKnet), Next Generation Attenuation-West 1 (NGA-West 1), and the Southern California Seismic Network (SCSN) in Various Distance and Magnitude Ranges

\begin{tabular}{|c|c|c|c|c|c|c|}
\hline & \multicolumn{2}{|c|}{ K-Net and KiK-net } & \multicolumn{2}{|c|}{ NGA-West 1} & \multicolumn{2}{|c|}{ SCSN } \\
\hline & $R<25 \mathrm{~km}$ & $R \geq 25 \mathrm{~km}$ & $R<25 \mathrm{~km}$ & $R \geq 25 \mathrm{~km}$ & $R<25 \mathrm{~km}$ & $R \geq 25 \mathrm{~km}$ \\
\hline $7 \leq M<8$ & 16 & 276 & 11 & 94 & 0 & 5 \\
\hline $6 \leq M<7$ & 94 & 1484 & 15 & 556 & 0 & 2 \\
\hline $5 \leq M<6$ & 433 & 5792 & 16 & 152 & 87 & 444 \\
\hline $4 \leq M<5$ & 0 & 0 & 12 & 55 & 569 & 5551 \\
\hline $3 \leq M<4$ & 0 & 0 & 0 & 0 & 2995 & 31,120 \\
\hline $2 \leq M<3$ & 0 & 0 & 0 & 0 & 4569 & 10,112 \\
\hline
\end{tabular}


(a)

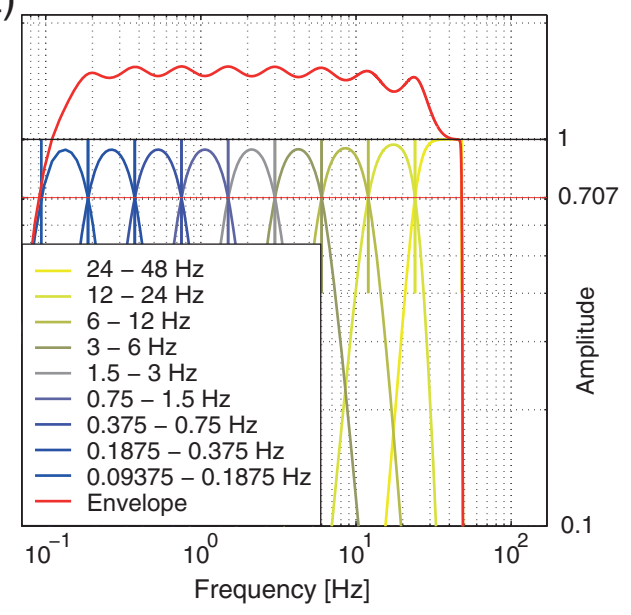

(b)

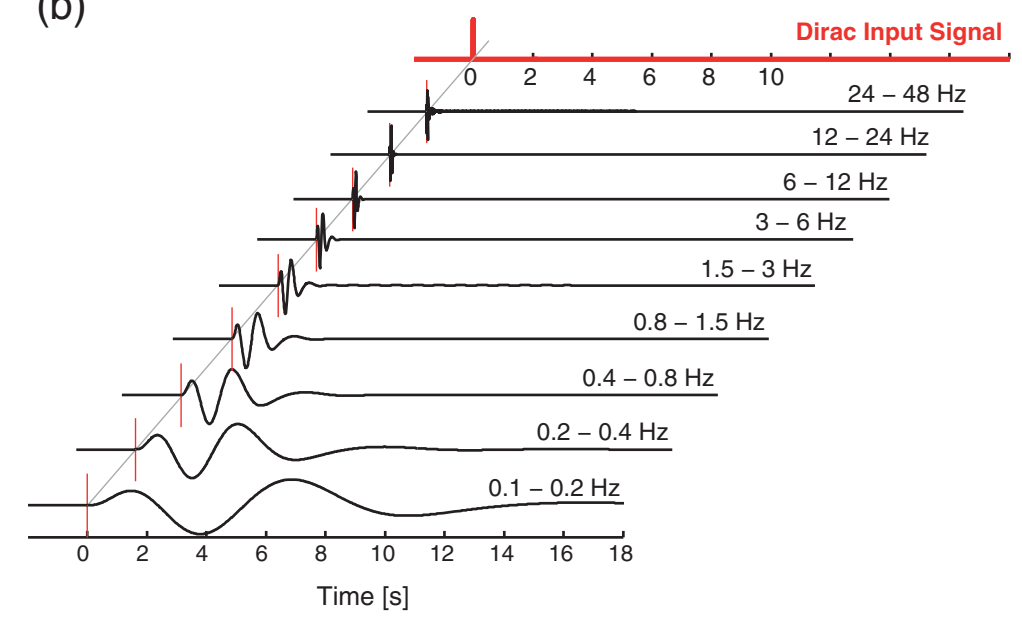

Figure 2. (a) Frequency and (b) impulse responses of the nine filter bank passbands.

based empirical approach. We term "target trace" the waveform for which we want to estimate the parameters; for each target trace, we use the $\mathrm{PGV}_{j}^{n b}(t)$ values of all other traces in the data set as training data, under exclusion of all traces that belong to the same event as the target trace.

\section{Bayesian Problem Statement}

Let $p\left[(M, R) \mid \mathrm{PGV}_{j}^{n b}(t)\right]$ denote the probability that an earthquake of magnitude $M$ at distance $R$ was the source of an observed vector of narrowband peak ground velocities, $\mathrm{PGV}_{j}^{n b}(t)$; this probability density function (PDF) is usually called the Bayesian posterior. Bayes theorem tells us that the joint posterior PDF for the two parameters is given by

$$
\begin{aligned}
p\left[(M, R) \mid \operatorname{PGV}_{j}^{n b}(t)\right]= & \frac{p\left[\operatorname{PGV}_{j}^{n b}(t) \mid(M, R)\right] p[M, R]}{p\left[\operatorname{PGV}_{j}^{n b}(t)\right]} \\
& \propto p\left[\operatorname{PGV}_{j}^{n b}(t) \mid(M, R)\right] p[M, R],
\end{aligned}
$$

in which $p\left[\mathrm{PGV}_{j}^{n b}(t) \mid(M, R)\right]$ is the probability of the observed PGV ${ }_{j}^{n b}(t)$, given the magnitude and the distance; this PDF is usually called the Bayesian likelihood. $p[M, R]$ is a probability model for seismicity as a function of magnitude and distance; this PDF is usually called the Bayesian prior. For the case of a single station observing seismicity with epicentral probabilities that are uniformly distributed in space and with magnitudes that are described by the GutenbergRichter relation (Cua, 2005):

$$
p[M, R] \propto p[M] p[R] \propto R 10^{-b M} .
$$

The $R$ term in equation (3) simply that reflects the area in a ring of width $d R$ grows as $R d R$. Equations (2) and (3) tell us that, given the observed ground-motion data $\operatorname{PGV}_{j}^{n b}(t)$, the most probable magnitude and distance (the posterior) is different from the magnitude and distance of an event that produces motions that best match $\mathrm{PGV}_{j}^{n b}(t)$ (the likelihood).
To clarify, let us assume a case in which the location of an event is already known and that all we want to do is determine the magnitude. Suppose we recorded $\operatorname{PGV}_{j}^{n b}\left(t^{\prime}\right)$ and this is the expected ground motion for an event having magnitude $M\left(t^{\prime}\right)$. That is, assume $\widehat{\mathrm{PGV}}_{j}^{n b}\left(t^{\prime}\right)=\left.\right|_{\max } p\left[\mathrm{PGV}_{j}^{n b}\left(t^{\prime}\right) \mid M\left(t^{\prime}\right)\right]$. For example, suppose we measured an amplitude of $100 \mathrm{~mm} / \mathrm{s}$ and that, at this distance, this is the amplitude expected to be produced by an $M 6.0$ event, according to the likelihood function. Given that we have just recorded this $100 \mathrm{~mm} / \mathrm{s}$ data, equations (2) and (3) tell us that the most probable magnitude is $<6.0$, say 5.6. That is because there is scatter in the relationship between magnitude and shaking amplitude, and smaller events are far more frequent than larger ones. In our imaginary case, if we measured $100 \mathrm{~mm} / \mathrm{s}$, then it is more likely that it is a larger than expected ground motion from one of several $M 5.6 \mathrm{~s}$ than it is an average record from an $M 6.0$ that occurs less frequently.

The distance prior and the magnitude priors work in opposite directions. The magnitude prior tells us that, given the shaking, a most probable magnitude is less than is predicted by a GMPE because small earthquakes are more numerous. The distance prior, on the other hand, implies that, given a recorded ground motion, the event is likely to be more distant than would be derived from a GMPE (just simple geometry); and, therefore, the magnitude is likely to be higher than what is expected from a GMPE.

In this study, we estimate the probabilities of the parameters $M$ and $R$ in an empirical way, based on a data set in which the data are - to first order-naturally distributed according to the prior distributions in equation (3) (Table 1). As we will see in the following section, these empirical probability estimates directly correspond to Bayesian posterior probabilities rather than Bayesian likelihoods.

\section{Magnitude Estimation}

We illustrate the parameter inference method in Figure 3, using a near-source record of the $2014 M_{\mathrm{w}} 6.0$ South Napa 
(a)

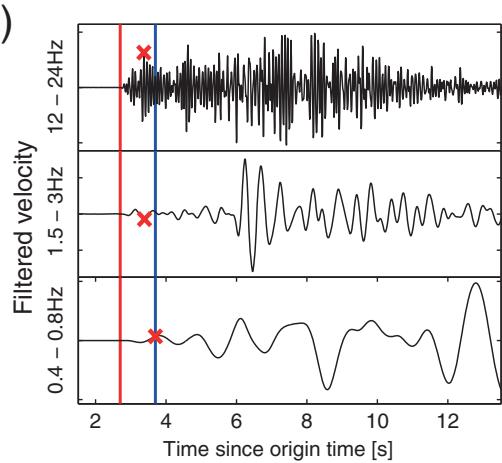

(c)

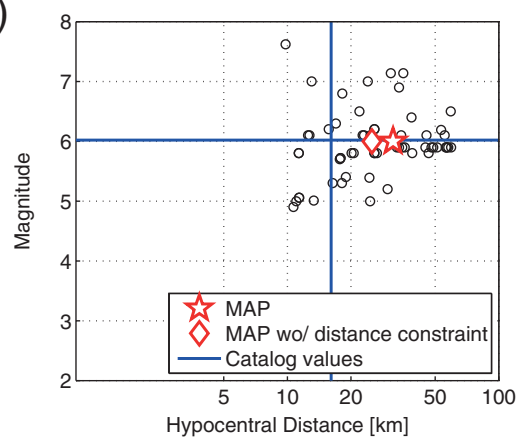

(b)

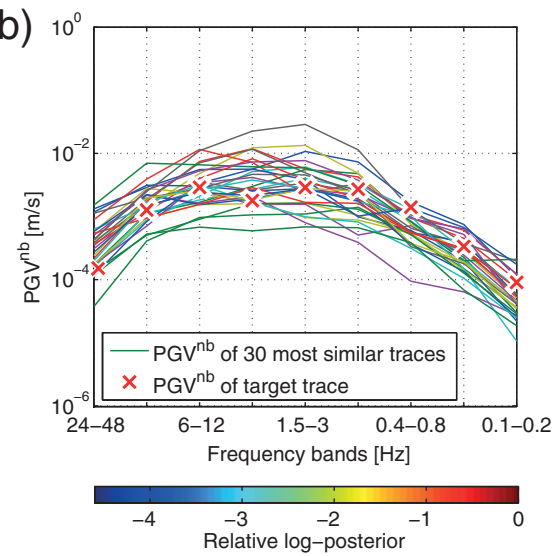

(d)

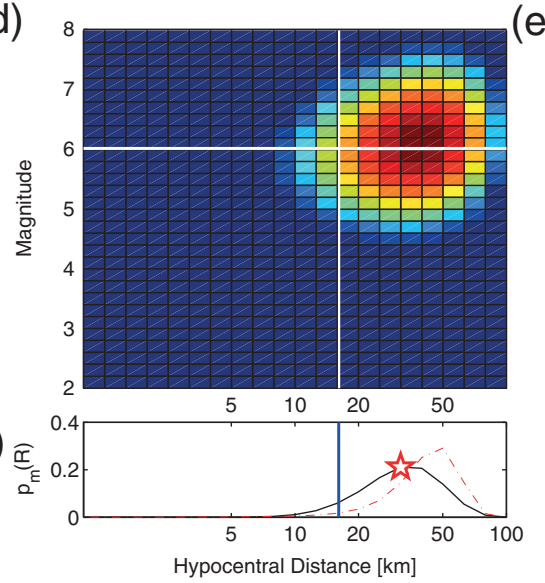

(e)

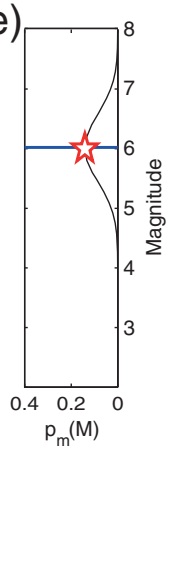

Figure 3. Parameter estimation scheme with the example of the vertical component of a near-source record ( $16 \mathrm{~km}$ hypocentral distance) of the $2014 M_{\mathrm{w}} 6.0$ South Napa earthquake, using $1 \mathrm{~s}$ of data: (a) three of the nine filtered velocity waveforms and peak amplitudes (PGV ${ }_{j}^{n b}$, red crosses) since the $P$-wave onset. The red and blue lines give the start and end of the used time window. (b) PGV $_{j}^{n b}$ of the South Napa record in all nine frequency bands (red crosses) and $\mathrm{PGV}_{i b}^{n b}$ values of the 30 most similar records in the data set (thin lines). (c) Magnitudes and hypocentral distances of those most-similar traces (black circles), earthquake catalog parameter values (blue lines), and most probable parameter estimates (maximum a posteriori [MAP]) obtained using only $\mathrm{PGV}^{n b}$ values (red diamond) and using an additional distance constraint (red star). (d) Bivariate relative log-posterior probability density function (PDF) fitted to the parameter values in (c). (e,f) Marginal distribution functions for magnitude and hypocentral distance, respectively, and MAP estimates (red stars). The dashed red line in (f) shows the additional distance constraint that was constructed as described in the Enhancing Magnitude Estimates with Additional Parameter Constraints section.

earthquake (hypocentral distance $16 \mathrm{~km}$ ) as an example target trace. We detect the $P$ arrival ("pick") for the target trace with a real-time picking algorithm. At any given time $t^{\prime}$ after the pick we observe $\mathrm{PGV}_{\text {target, } j}^{n b}\left(t^{\prime}\right)$ (Fig. 3a). We then scan the training data set to identify the $n$ traces that at time $t^{\prime}$ had the most similar $\operatorname{PGV}^{n b}\left(t^{\prime}\right)$ values (Fig. $3 \mathrm{~b}$ ); $n$ is a free parameter, and we use $n=30$. We define similarity as the squared difference between the log amplitudes of target and training traces, summed over the $j=1, \ldots, f$ frequency bands:

$$
\begin{aligned}
\Delta \operatorname{PGV}_{i}^{n b}\left(t^{\prime}\right)= & \sum_{j=1}^{f}\left(\log _{10}\left[\operatorname{PGV}_{\text {target }, j}^{n b}\left(t^{\prime}\right)\right]\right. \\
& \left.-\log _{10}\left[\operatorname{PGV}_{i, j}^{n b}\left(t^{\prime}\right)\right]\right)^{2},
\end{aligned}
$$

in which $i$ is the trace index of the training data set. We then use the magnitudes $M_{t r}$ and hypocentral distances $R_{t r}$ of the identified most-similar training traces (Fig. 3c) to construct the probability function for the EEW parameters of the target record. Note that we identify the $n$ most similar traces for vertical and horizontal traces separately; $M_{t r}$ and $R_{t r}$ are hence vectors of lengths $2 n$. We approximate the distribution of the resulting set $\left[M_{t r}, R_{t r}\right]$ with a coarsely discretized bivariate Gaussian density function $p\left(M, \log _{10}[R] \mid \mathrm{PGV}_{\text {target }, j}^{n b}\left(t^{\prime}\right)\right) \sim \mathcal{N}(\mu, \boldsymbol{\Sigma})$, in which $\mu=\left[\left\langle M_{t r}\right\rangle,\left\langle\log _{10}\left[R_{t r}\right]\right\rangle\right]^{T}$ are the mean magnitudes and logarithmic distances of the set and $\boldsymbol{\Sigma}$ is the corresponding covariance matrix (Fig. 3d).

We use the resulting $p\left(M, \log _{10}[R] \mid \mathrm{PGV}^{n b}\left(t^{\prime}\right)\right)$ as the empirical PDF of the EEW parameters of the target record and maximize it to find the maximum a posteriori estimates $\hat{M}_{\text {map }}$ and $\hat{R}_{\text {map }}$. Furthermore, we compute univariable marginal probability functions $p_{m}\left(M\left(t^{\prime}\right)\right)$ and $p_{m}\left(\log _{10}\left[R\left(t^{\prime}\right)\right]\right)$ by numerically integrating out either the distance or the magnitude parameter from the bivariate PDF. The maxima of the bivariate and of the marginal PDFs are equivalent.

In summary, we are asking the question: Given a recorded $\mathrm{PGV}_{j}^{n b}(t)$, what is the most frequent $(M, R)$ combination in a catalog of records with similar $\operatorname{PGV}_{j}^{n b}(t)$ values? 
If the catalog contained data that were distributed uniformly in magnitude and distance (i.e., $p[M, R]=$ constant), then, according to equation (2), our procedure would reveal the likelihood function. That is, in this unrealistic case, $p\left[(M, R) \mid \mathrm{PGV}_{j}^{n b}(t)\right]=p\left[\mathrm{PGV}_{j}^{n b}(t) \mid(M, R)\right]$. Our procedure would then be analogous to determining a GMPE for our filter bank values. However, because we applied only minimum selection criteria for compiling the data set, such as maximum distance and minimum magnitude cut-offs, the data are approximately represented according to their natural distribution; as a consequence, the priors in equation (3) are an integral part of the estimation procedure. In terms of the example from the previous section in which the $100 \mathrm{~mm} / \mathrm{s}$ observed ground-motion velocity was more likely from aboveaverage records of one of the several $M 5.6$ events than from an average record of an $M 6.0$ event, when we search the data set for the $n$ most similar records, we are more likely to find those above-average $M 5.6$ records because there are more of them in the data set than $M 6.0$ records. Likewise, records with large source-station distances are naturally overrepresented in the data set relative to records with short distances, in agreement with equation (3). Our empirical parameter estimation procedure therefore implicitly considers both the magnitude and the distance prior distributions and directly returns the desired posterior distribution function.

\section{Combining Magnitude Estimates from Multiple Stations}

Combining magnitude estimates from multiple stations is straightforward because of the probabilistic formulation of the algorithm: the joint marginal magnitude probability function is obtained by multiplying single-station marginal probabilities:

$$
p_{m}^{k}(M, t)=\prod_{s=1}^{k} p_{m}^{s}(M, t),
$$

in which $p_{m}^{s}(M, t)$ is the marginal magnitude probability function of the $s$ th out of $k$ stations. Stations that have longer snippets of available data because they are closer to the epicenter will tend to have narrower marginal probability functions and therefore have stronger influence on the joint maximum a posteriori estimate $\hat{M}_{\text {map }}$.

Enhancing Magnitude Estimates with Additional Parameter Constraints

The obtained magnitude estimates $\hat{M}_{\text {map }}$ are entirely based on the transient frequency content of the waveforms and do not require a hypocenter location. However, if other independent constraints on either of the two parameters are available, they can be combined with the estimates in much the same way that we combine estimates from multiple stations (equation 5). There are numerous candidate constraints that could be used in this sense: parameter estimates from other EEW algorithms that run in parallel, distance estimates from a real-time location algorithm (e.g., based on the con- cept of not-yet-arrived data; Zhou, 1994; Cua and Heaton, 2007; Satriano et al., 2008), $S-P$ onset times $t_{S-P}$, the waveform-based distance estimation method of Odaka et al. (2003), or distance priors from seismicity observations (e.g., Kagan and Knopoff, 1981; Meier et al., 2014) and from proximity to mapped faults. Because distance and magnitude uncertainties are inherently coupled, any constraint that reduces the distance uncertainty will also reduce magnitude uncertainties. A schematic illustration of the parameter inference framework with multiple stations, additional independent constraints, and prior information is shown in Figure 4.

In a full EEW system, increasingly well-constrained distance estimates would become available over time through a real-time event location algorithm, helping to reduce magnitude uncertainties. For this study, however, we neither computed such additional distance constraints nor relocated the processed events. Instead, we simulate the distance constraints and show what effect they have on the magnitude estimations. All results from this study are shown with and without using such additional constraints.

To imitate realistic distance constraints, we construct a distance PDF $p\left(\log _{10}[R]\right)$ by perturbing the catalog hypocentral distance of the target record with a perturbation $\Delta R$ that is randomly sampled from a normal distribution. The width of the distribution depends on the number of triggered stations: for magnitude estimates of which less than three triggered stations are available, we use $\sigma_{R}=20 \mathrm{~km}$. If three or more stations are used for the estimation, we set $\sigma_{R}=10 \mathrm{~km}$. The distance PDF $p(R)$ is then constructed as $p(R) \sim \mathcal{N}\left(\mu_{R}, \sigma_{R}^{2}\right)$, in which $\mu_{R}=R_{\text {catalog }}+\Delta R$ is the perturbed center of the distribution. We transform this function $p(R)$ into the log domain to obtain $p\left(\log _{10}[R]\right)$, multiply it with the bivariate parameter PDF (Fig. $3 \mathrm{~d}$ ), and use the resulting updated bivariate PDF to estimate $\hat{M}_{\text {map }}(t)$ via the marginal magnitude PDF. The chosen values for $\sigma_{R}$ of 20 and $10 \mathrm{~km}$ represent conservative estimates; they were chosen to avoid artificially increasing the algorithm performance.

\section{Performance of the Real-Time Parameter Inference}

To evaluate the performance of the algorithm at different stages of an ongoing event, we define the time-dependent magnitude residual $M_{\text {rsd }}(t)=M_{\text {catalog }}-\hat{M}_{\text {map }}(t)$, in which $\hat{M}_{\text {map }}(t)$ is the time-dependent maximum a posteriori magnitude estimate and $M_{\text {catalog }}$ is the reported catalog magnitude. In the following section, we analyze these residuals and how they change as increasing amounts of data become available over time.

\section{Parameter Inference with Data from 1 Station}

The first parameter estimation can be made as soon as the first station has recorded $0.5 \mathrm{~s}$ of data since the onset of the direct $P$ phase. The left column of Figure 5a,c,e, and g shows $M_{\text {rsd }}(t)$ from single-station parameter estimates for all 64,460 
(a) $3^{\text {rd }}$ Station
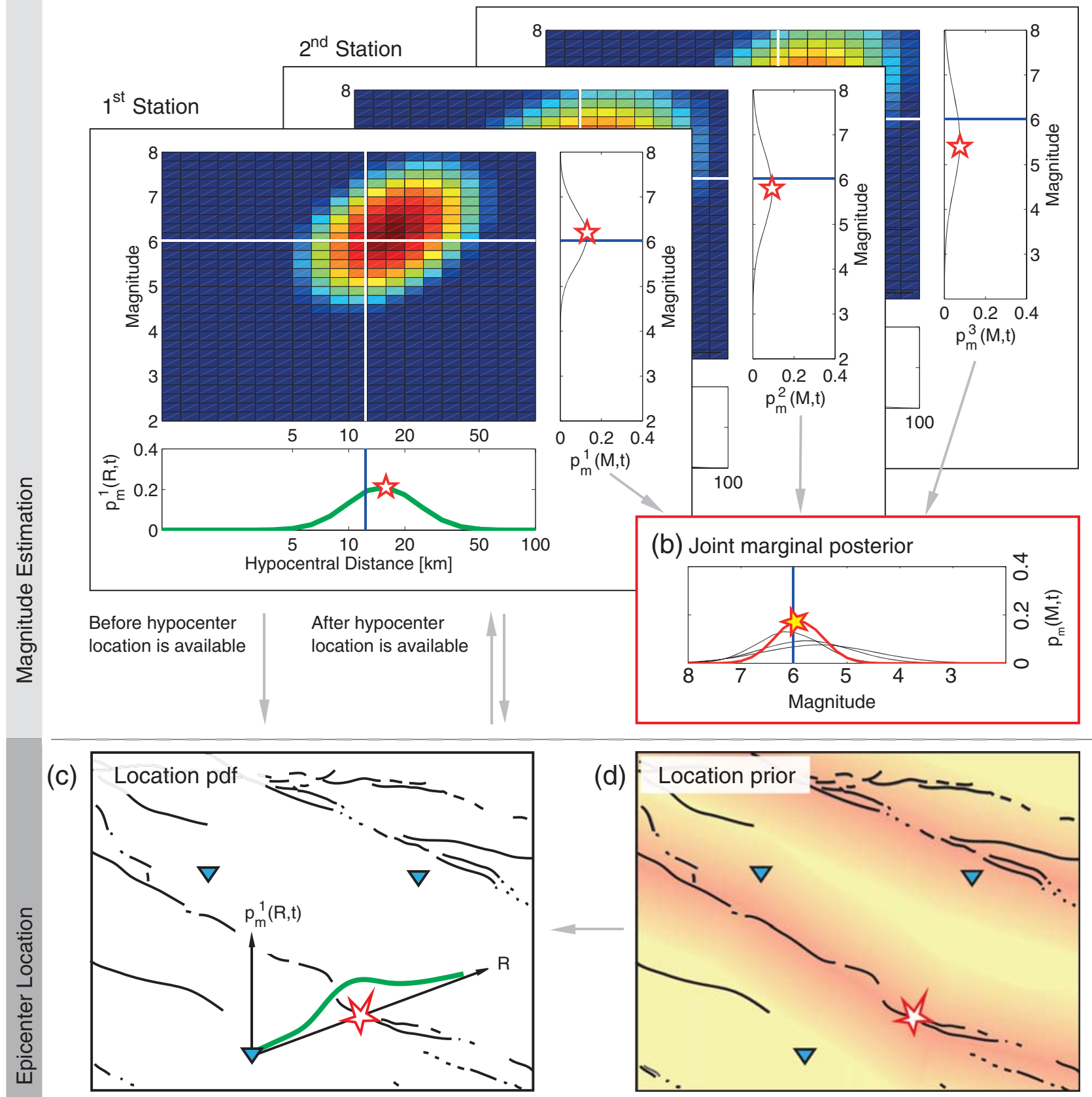

Figure 4. Schematic illustration of how the parameter estimates of the Gutenberg algorithm (GBA) can be embedded in a full probabilistic earthquake early warning system. The gray arrows represent information flow. (a) For each triggered station, the GBA produces a magnitude and a distance PDF. (b) The magnitude PDFs can be combined to obtain the joint marginal posterior magnitude PDF. (c) Before an independent hypocenter location estimate (star) is available, the GBA distance PDFs may be used for a rough early location estimate. As soon as an early standard location estimate is available, the corresponding source-station distances can in turn be used to update the bivariate parameter PDF and thereby improve the magnitude estimates. At any stage, prior information (e.g., based on fault proximity models [d]) may be used to improve the estimates.

records of the data set as a function of catalog magnitude for $t=0.5,1,3$, and $10 \mathrm{~s}$ after the $P$ onset. For the entire data set, the mean residual is $0.03 \pm 0.51$ with $1 \mathrm{~s}$ of data from one station, indicating that information from the first second of a single record is diagnostic of the event magnitude. However, there is a clear magnitude saturation for events with $M>6$, and records with $M<3$ have a bias toward magnitude overestimation. As the record length increases, the distribution of $M_{\text {rsd }}$ becomes only slightly narrower $(0.01 \pm 0.47$ at $3 \mathrm{~s})$, and the underestimation of large events persists. The overestimation at low magnitudes is produced by a set of records with very short hypocentral distances. For such records, the algo- 


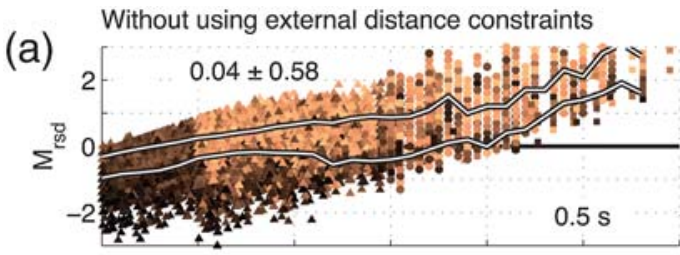

(c)
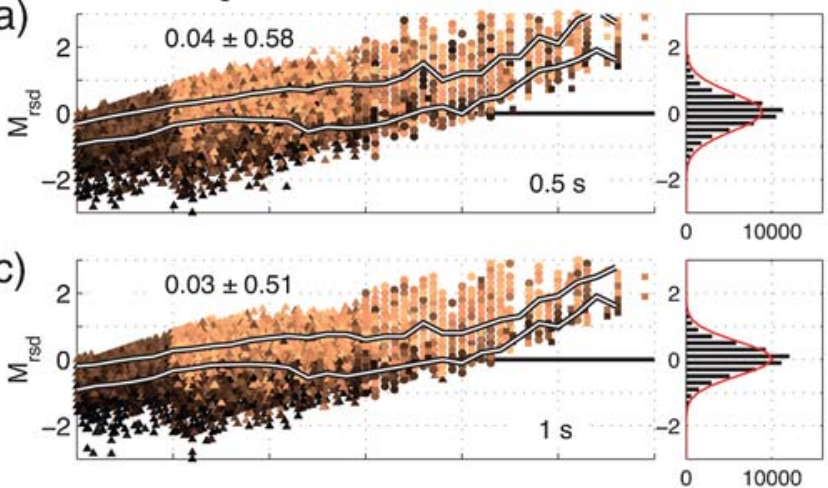

(e)
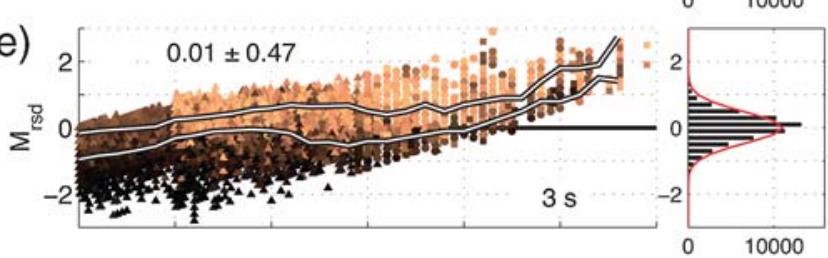

(g)

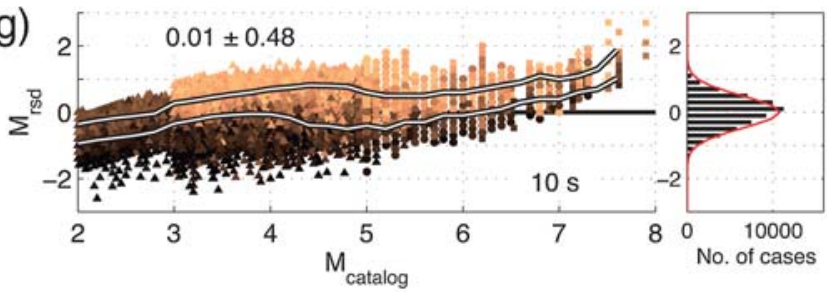

(b)

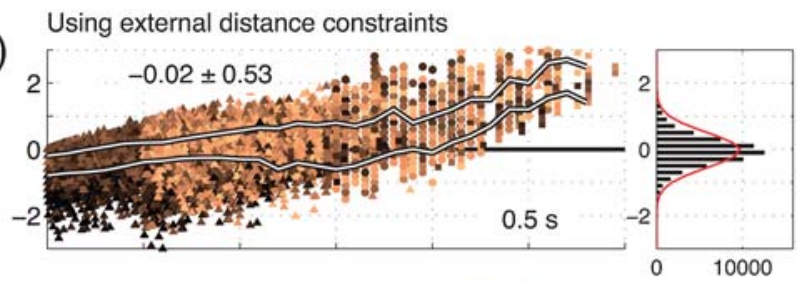

(d)

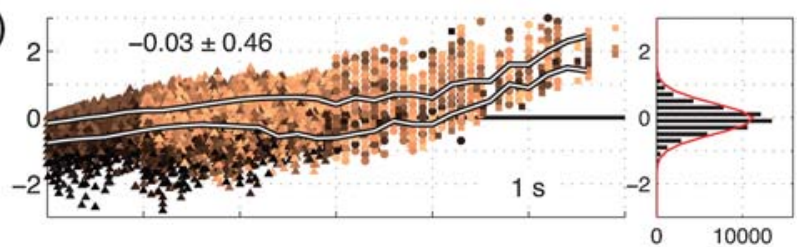

(f)

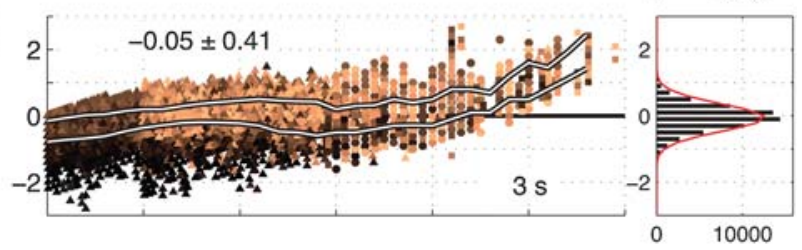

(h)

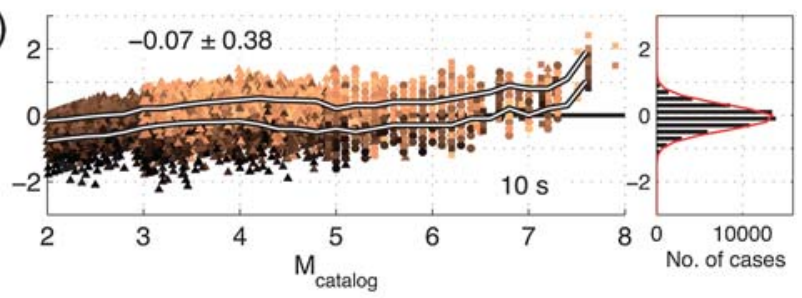

Hypocentral distance $[\mathrm{km}]$

Figure 5. Magnitude residuals from single-station estimates for all 64,460 records of the data set using $0.5,1,3$, and $10 \mathrm{~s}$ of data based on $\mathrm{PGV}^{n b}(t)$ values, without additional distance constraints (left column) and with additional distance constraints (right column). The color represents the catalog hypocentral distance for each record. Because of the large number of data points, many points are overprinted. The white lines give the 16th and the 84th percentiles $(1 \sigma)$ evaluated in 0.2 magnitude unit bins to give a more concise impression of the distribution.

rithm tends to overestimate the distance and, as a consequence, overestimates the magnitude. Although the overall scatter is relatively low, there are substantial numbers of records with high absolute residuals of $>1$ magnitude unit. A similar behavior has also been reported for the single-station onsite algorithm (Kanamori, 2005). Such misclassifications could translate into false alerts in an operational EEW system.

The saturation magnitude increases with increasing record length. The saturation reflects the fact that the $\mathrm{PGV}^{n b}$ values of events with $M>6.5$ at, say, $3 \mathrm{~s}$ are not significantly higher than those of an $M \sim 6.5$ event. It is only when much longer time windows are available that the algorithm can accurately classify $M 7+$ records. For events with $M>7.5$, even $10 \mathrm{~s}$ are not long enough, suggesting that the rupture process is still ongoing for events of such high magnitudes.

If we assume that additional distance constraints are available (compare with the Enhancing Magnitude Estimates with Additional Parameter Constraints section), the bias issues exhibited by the estimates solely based on $\operatorname{PGV}^{n b}(t)$ are reduced (right column in Fig. 5b,d,f,h), and the overall distribution becomes narrower $(-0.05 \pm 0.41$ with $3 \mathrm{~s}$ of data); here we used $\sigma_{R}=20 \mathrm{~km}$ for simulating the independent distance constraint.

Parameter Inference with Data from 2 to $n$ Stations

In densely instrumented areas, multiple stations typically record the $P$-wave onset before the first station has recorded more than $4 \mathrm{~s}$ (Kuyuk and Allen, 2013). A majority of estimates in Figure 5 therefore, in fact, are not relevant for EEW because at the time they become available, more proximal stations will already have triggered the estimations. In Figure 6, we present the $M_{\text {rsd }}(t)$ as they would be computed in a real-time EEW system, using only the most proximal records for each earthquake. At first only the nearest station to the epicenter provides data until, over time, data from additional stations become available and influence the estimates. We compute $M_{\mathrm{rsd}}(t)$ at the instant when the first, second, 

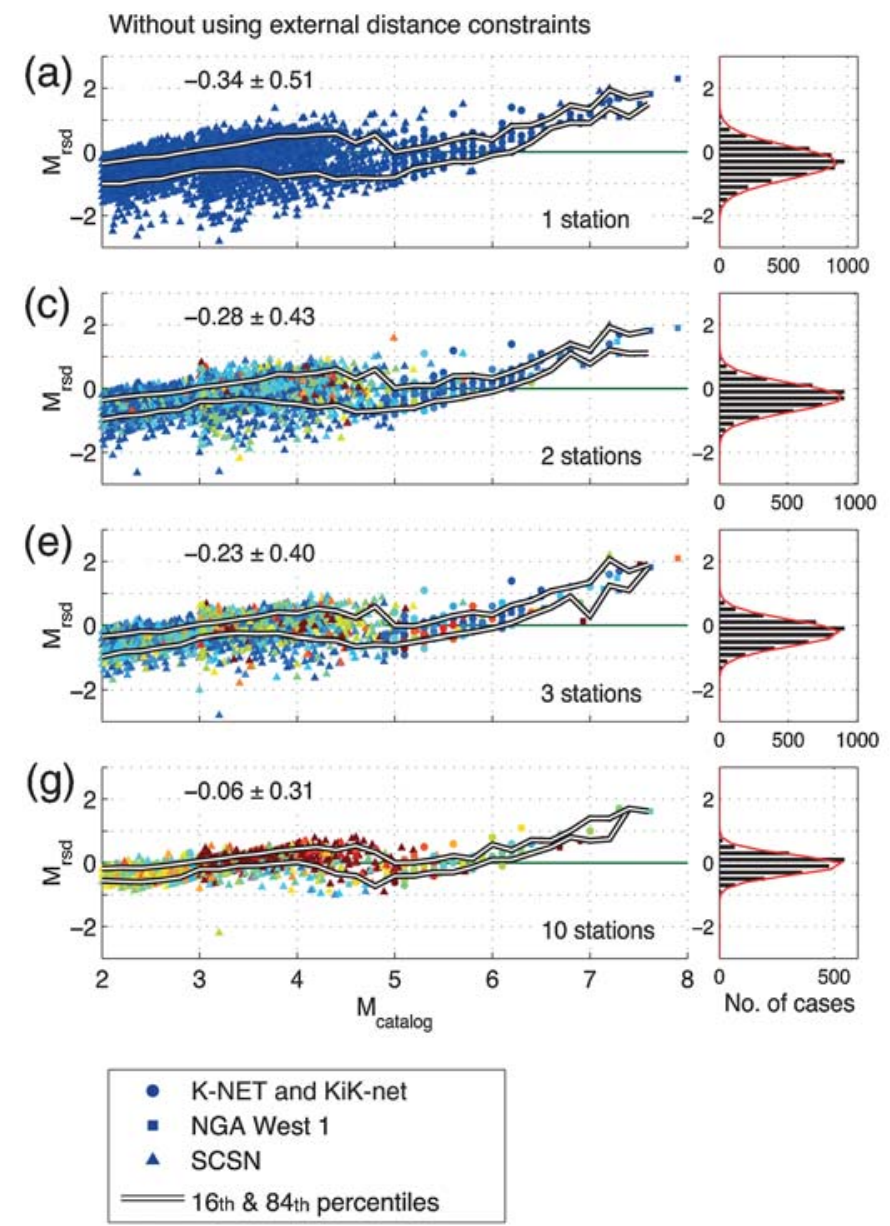
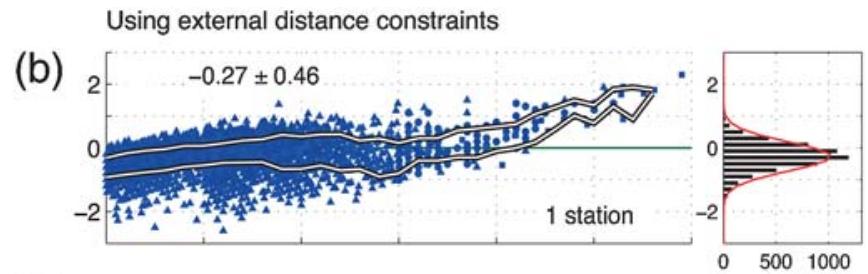

(d)

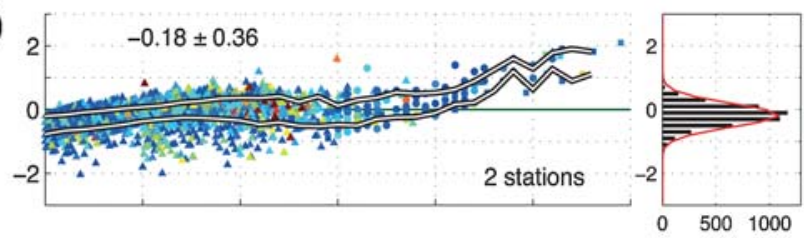

(f)

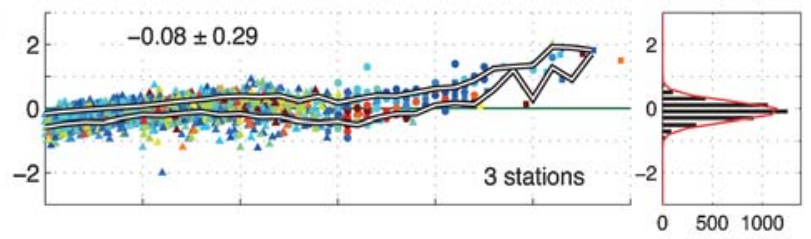

(h)
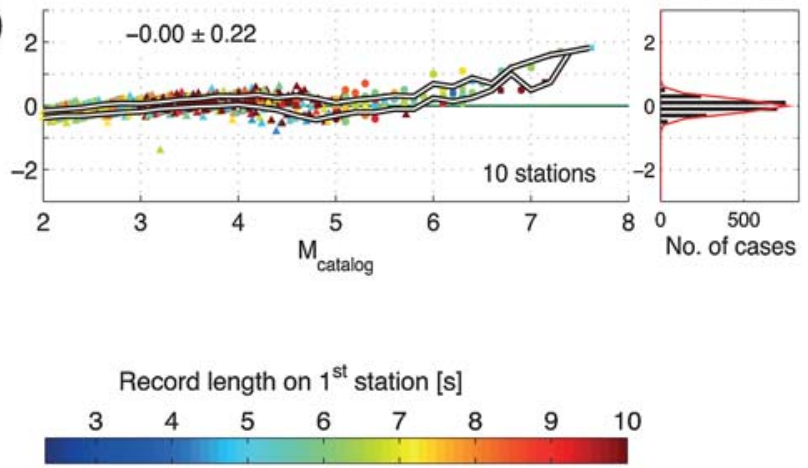

Figure 6. Magnitude residuals for all 5852 events of the data set when the first, second, third, and tenth stations have recorded $1 \mathrm{~s}$ since the $P$-wave onset, based solely on the observed $\mathrm{PGV}^{n b}$ values (left column) and using an additional distance constraint (right column). Histograms show the distribution of residuals over the entire magnitude range. The color indicates the amount of data that was available on the first station at the time of parameter estimation. The white lines give the 16th and the 84th percentiles in 0.2 magnitude unit bins. The events are not shown if their total number of records is smaller than the number of records used in a subfigure. For example, there are only three records of the $M_{\mathrm{w}} 7.9$ Denali, Alaska, earthquake, so this event no longer appears on subplots (g) and (h).

third, and tenth stations have recorded $1 \mathrm{~s}$ of data following the $P$ onset. From the more proximal stations, we use whatever data are available at that instant. For the multistation estimates, we use equation (5) to obtain the joint marginal posterior magnitude distribution and $\hat{M}_{\text {map }}(t)$.

Figure 6 reveals that with the most proximal records alone, only a small number of events are strongly misclassified. Using $1 \mathrm{~s}$ of data from the second station (Fig. 6c), 4.6\% of events have absolute magnitude residuals $\left|M_{\text {rsd }}\right|>1$, a majority of which are events with catalog magnitudes $M_{\text {catalog }}<3$. Furthermore, combining two stations slightly reduces the scatter to $-0.28 \pm 0.43$ magnitude units (using $1 \mathrm{~s}$ on the second station). Adding the independent distance constraints for both stations decreases the scatter to $M_{\text {rsd }}$ to $-0.18 \pm 0.36$. Although the average bias was negligible for all single-station estimates (Fig. 5), the bias for the proximal records lies between $\sim-0.1$ and $\sim-0.35$ when small numbers of stations are used. This is caused by the biased $M<3$ events, which have higher relative weight in this statistic than in Figure 5, because the numerous (unbiased) far-source records of the higher magnitude events are not included here. For the records with $M \geq 3$, the mean residuals are 0.1 and 0.07 with data from the second and third stations, respectively.

As soon as data from two stations are available, the distance estimates $\hat{R}_{\text {map }}$ obtained from maximizing the marginal distance PDFs could be used to roughly constrain the epicenter. Because the $\mathrm{PGV}^{n b}(t)$ values are independent of the $P$-onset times, the four observations (two $\mathrm{PGV}^{n b}$-based distance estimates and two $P$-onset times) might be enough for an early location estimate. However, this exercise has not yet been conducted and would go beyond the scope of this article.

Once three or more stations have triggered, the EEW parameters $M$ and $R$ become increasingly better constrained. Furthermore, at this stage, enough $P$-phase arrival times are available to make rough estimations of the epicenter using an equal differential time norm location scheme (Zhou, 1994; Satriano et al., 2008). If we include the additional 
distance constraint using $\sigma_{R}=10 \mathrm{~km}$ (right column of Fig. $6 \mathrm{~b}, \mathrm{~d}, \mathrm{f}, \mathrm{h})$, the residual distribution is very narrow $(-0.08 \pm 0.29$ with $1 \mathrm{~s}$ of data from the third station), the overestimation of small-magnitude events with near-source recordings becomes negligible, and the number of strongly misclassified events amounts to only $\sim 0.6 \%$ of cases. The underestimation of large magnitudes, however, persists even when the location is well constrained.

\section{Discussion}

To provide EEW alerts at proximal sites to the epicenter, alerts need to be based on less data from fewer stations than what can be used for alerts at more distant target sites. However, using less data is expected to translate into larger parameter uncertainties. The Gutenberg magnitude estimation algorithm minimizes these uncertainties by maximally exploiting the available data, and it systematically quantifies them.

The algorithm is based on the principle that ground-motion amplitudes at different frequencies are, to first order, governed by the event magnitude and the source-station distance, which are the two key parameters needed for EEW. Consequently, observed amplitudes can in turn be used to infer these parameters. In any individual frequency band, there is a trade-off between the two EEW parameters in that an observed amplitude can be caused by a large event at a large distance or by a comparatively smaller event at a smaller distance. The GBA resolves this trade-off by jointly inferring the two parameters from multiple frequency bands: high (relative) amplitudes at high frequencies are only observed if the source-station distance is short; high (relative) amplitudes at low frequencies are only observed if the magnitude is high. By requiring similarity of amplitudes in all frequency bands (equation 4), the algorithm finds the unique distance-magnitude combination that is in agreement with the observations across all frequencies. We chose the name "Gutenberg algorithm" because the practice of inferring earthquake location and magnitudes from different frequency bands of a single record resembles the habit of the late Beno Gutenberg, who would use numerous recording instruments from the same site in Pasadena, each of which was sensitive to a specific frequency range, to analyze teleseismic events from around the globe. The algorithm relies only on the frequency content of the real-time waveforms. It does not require additional information such as an epicenter location estimate. Because of its probabilistic formulation, however, any form of independent constraint on event magnitudes and source-station distances is readily incorporated. The probabilistic formulation furthermore facilitates the natural evolution from an onsite- to a regional-type algorithm. Event characterizations are started as soon as the first station has triggered and are then perpetually updated as more information becomes available. The continuous uncertainty quantifications that come with every parameter estimate allow end users to decide whether or not a certain damage mitigation action should be initiated.

Although this parameter inference scheme requires more computational effort than a $\tau_{p}^{\max }$ - or $P_{d}$-based inversion, it is feasible in real time. The processing of $1 \mathrm{~s}$ of a single waveform through the filter bank takes on average $5 \times 10^{-4} \mathrm{~s}$ on a MacBook Pro laptop with a $2.5 \mathrm{GHz}$ Core i7 processor. With a training data set of $\sim 190,000$ preprocessed training waveforms, the parameter estimation with two horizontal and one vertical target traces takes $0.02 \mathrm{~s}$ on the same processor, that is, updates at a rate of $0.5 \mathrm{~s}$ are possible.

The performance of the algorithm depends mainly on the amount of data available at a given time and on the magnitude of the target event. Over the entire magnitude range, the parameter estimates reach higher precision at earlier times than existing algorithms, although for most algorithms only a little information on estimation uncertainties is typically published. Brown et al. (2011) provide detailed uncertainty estimations and report similar residual distributions for the single-station estimates $(-0.1 \pm 0.5$ using $3 \mathrm{~s}$ of data from the first station, versus $-0.01 \pm 0.47$ from the GBA). When information from multiple stations are combined, the residuals of Brown et al. (2011) are not reduced substantially (e.g., $-0.1 \pm 0.5$ using $3 \mathrm{~s}$ of data from the first four stations), whereas the corresponding Gutenberg estimation residuals reduce to $-0.04 \pm 0.25$ with the same amount of data (not shown). However, the studies are not directly comparable because they used different data sets.

For all estimations, a distinct magnitude saturation is observed, in agreement with what has been reported by Rydelek et al. (2007). The onset of magnitude saturation depends on the time-window length that was used for the estimation. For windows $<4 \mathrm{~s}$, saturation starts at $M \sim 6.5$. Higher magnitudes are estimated accurately only when longer time windows are used. We interpret this as an indication that amplitude-based EEW magnitude estimates are not in fact predictive, but observational. A magnitude can be recognized (and accurately estimated) if the maximum of the source time function has already been reached and is mapped into the portion of the seismogram that is used to make the estimation. This is an important observation in the context of an ongoing debate on whether earthquake rupture is a deterministic process (Ellsworth and Beroza, 1995; Olson and Allen, 2005; Colombelli et al., 2014) or not (Rydelek and Horiuchi, 2006; Rydelek et al., 2007). The results from this study support the nondeterministic case. This has important consequences for EEW in that, depending on the length of the time windows used, high magnitude estimates have to be considered minimum estimates until more data become available. The upside is that whether or not an estimate is a minimum estimate can be evaluated from the lengths of the available data snippets.

Furthermore, for events with $M$ above the saturation magnitude of $\sim 6.5$, EEW algorithms are faced with another issue: the finiteness of the source mechanism as well as the complexities of slip distributions become important for 


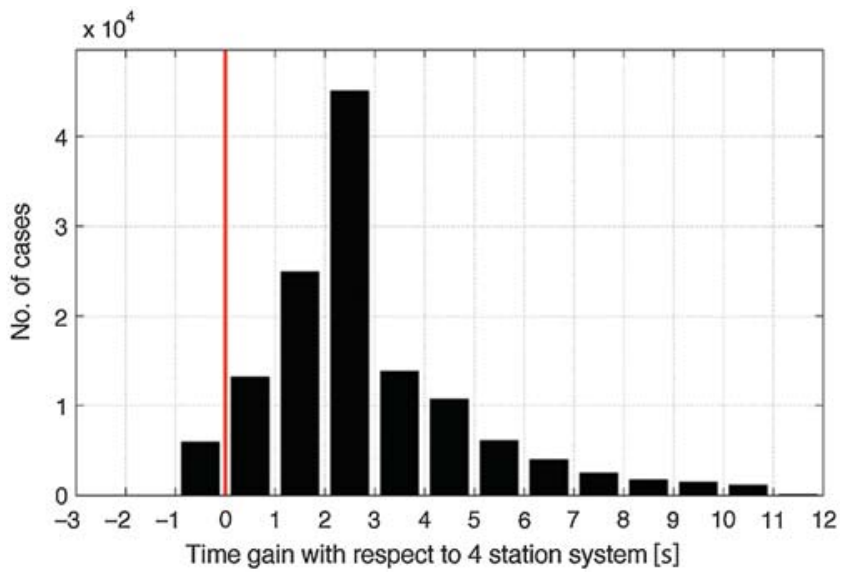

Figure 7. Distribution of time gain if first alerts were issued when the second-triggering station has recorded $1 \mathrm{~s}$, relative to when alerts are issued once the fourth station has triggered.

ground-motion prediction. Several EEW algorithms have been proposed to resolve these complexities (e.g. Böse et al., 2012; Crowell et al., 2012; Minson et al., 2014) and show promising performance in offline tests. However, because they either use data from numerous stations or strong ground motions as input data, their parameter estimates become available with considerable delay when the strong shaking has already begun at proximal sites. These algorithms are therefore most relevant for more distant target sites. Note that for the proximal sites, minimum magnitude estimates may be enough to provide useful EEW alerts.

Alerts based on observations at a single near-source station may only be suitable for EEW applications with very low costs of false alerts. With such little data, magnitude residuals are relatively high and the number of strong misclassifications may be intolerable. However, many of the misclassifications presumably can be filtered out in an operational EEW system, for example, with an absolute amplitude threshold or with other waveform-based criteria (e.g., Böse, Hauksson, Solanki, Kanamori, Wu, and Heaton, 2009). As soon as a second station has recorded 1 or 2 s, on the other hand, there are only few near-source overestimations, and the magnitude residuals have by then dropped to $-0.28 \pm 0.43$.

If EEW alerts could be based on two rather than on four or six stations, the size of the blind zones could be reduced substantially. Figure 7 shows the time gain distribution in southern California if EEW alerts can be issued when the second station has recorded $1 \mathrm{~s}$, relative to the hypothetical system of Kuyuk and Allen (2013), which requires 4 s on the first station and a $P$-wave trigger at the fourth nearest station. The time gains have been evaluated on a grid across California, assuming a data transmission and processing delay of $4 \mathrm{~s}$, uniform hypocentral depths of $8 \mathrm{~km}$, and seismic velocities $V_{P}=6.5 \mathrm{~km} / \mathrm{s}$ and $V_{S}=3.4 \mathrm{~km} / \mathrm{s}$. Only sites that have a station within $100 \mathrm{~km}$ were used. The median time gain is $2.5 \mathrm{~s}$, and some locations can benefit from a gain of up to $10 \mathrm{~s}$. Although a few seconds may not seem like a large gain, in these sites they determine whether a site falls within the blind zone (i.e., gets no warning) or not; $2.5 \mathrm{~s}$ can reduce the blind zone radius by $\sim 8 \mathrm{~km}$ for shallow events. However, the lead time at proximal sites to the epicenter will always be short (several seconds at most). Correspondingly, the saturation magnitudes will be relatively low and ground motions may be underestimated if the event magnitude is higher than the saturation magnitude. However, for these proximal sites, waiting for more data to become available is not an option.

If EEW alerts are to be based on two records, a highly reliable event associator is necessary to complement the parameter estimation algorithm. Kuyuk et al. (2013) convincingly demonstrated how a well-selected set of logic criteria can drastically reduce misclassifications (e.g., false alerts). Presumably, even stronger performance could be obtained if the classification was based on the frequency content of observed real-time waveforms (e.g., Beyreuther and Wassermann, 2011; Hammer et al., 2012; Wu et al., 2015). Furthermore, in its current formulation, the GBA is limited to areas with predominantly shallow crustal seismicity. For other tectonic settings where focal depths may play a more important role, it would need to be adapted. The modular design of the data set makes it very easy to add more data from a region of interest, for example, European data. Finally, because the GBA at least partly uses different data than existing EEW algorithms, combining its estimates with those of other algorithms to create ensemble estimations may lead to an improvement of the parameter estimates. Because of the probabilistic formulation of the algorithm, this would be straightforward to do.

\section{Conclusions}

During most damaging earthquakes, destructive groundmotion amplitudes are concentrated at proximal sites around the epicenter. EEW for proximal sites, however, is a challenge because the onset of strong ground motion occurs shortly after the $P$-wave onset at the closest stations. Consequently, only the earliest EEW estimates are available in time. We presented a probabilistic algorithm that minimizes the uncertainties of these earliest warnings, systematically quantifies them, and that naturally evolves from an onsite- into a regional-type EEW algorithm (with correspondingly lower uncertainties). The GBA jointly estimates event magnitude and source-station distances of the first records directly from the observed waveform frequency content and therefore does not need to wait until an early epicenter location estimate becomes available. Once a location estimate is available, the algorithm facilitates the systematic incorporation of this information to improve the magnitude estimates. The GBA reaches magnitude residuals of $-0.01 \pm 0.47$ with only $2 \mathrm{~s}$ of data on a single station, a level of estimation precision that has been reported for existing algorithms with data from four or more stations. However, information from a second 
station has to be incorporated to prevent occasional strong misclassifications $\left(\left|M_{\mathrm{rsd}}\right|>1\right)$. The median time gain of such two-station warnings with respect to warnings from a system requiring four stations is on the order of $2-3 \mathrm{~s}$ in California. Although this is only a short time, these seconds are crucial for proximal sites to the epicenter in that they may determine whether a site falls within or outside the blind zone. Warning times at proximal sites, however, will always be short —on the order of single seconds-and allow only a limited set of potential damage mitigation actions. Furthermore, the magnitude estimates saturate above a threshold. The onset of saturation is observed to be a function of the signal length that is used for the estimation. Depending on how much data is available during an ongoing event, magnitude estimates have to be considered minimum estimates. This observation further suggests that amplitude-based EEW magnitude estimates are observational, rather than predictive. A given magnitude can only be accurately estimated once the bulk of the energy release has been mapped into the seismograms that are used for the estimation. This finding supports the interpretation of earthquakes as a nondeterministic phenomenon.

\section{Data and Resources}

The Japanese waveform data were obtained from http:// www.kik.bosai.go.jp/ (last accessed August 2013). The Next Generation Attenuation-West 1 waveform and metadata were obtained from http://peer.berkeley.edu (last accessed March 2014). The southern California waveform, catalog, and arrival time data were obtained via the Seismic Transfer Program tool from http://scedc.caltech.edu/research-tools/ stp-index.html (last accessed September 2015). The SBPxalgorithm was published as SBPx.m on mathworks.com/ matlabcentral/fileexchange/ (last accessed July 2015).

\section{Acknowledgments}

We would like to thank Yannik Behr, Carlo Cauzzi, Maren Boese, and Julius Smith for valuable discussions and comments.

\section{References}

Allen, R. M., and H. Kanamori (2003). The potential for earthquake early warning in southern California, Science 300, no. 5620, 786-789.

Allen, T. I., D. J. Wald, and C. B. Worden (2012). Intensity attenuation for active crustal regions, J. Seismol. 16, no. 3, 409-433.

Baer, M., and U. Kradolfer (1987). An automatic phase picker for local and teleseismic events, Bull. Seismol. Soc. Am. 77, no. 4, 1437-1445.

Beyreuther, M., and J. Wassermann (2011). Hidden semi-Markov model based earthquake classification system using weighted finite-state transducers, Nonlinear Process. Geophys. 18, no. 1, 81-89.

Böse, M., E. Hauksson, K. Solanki, H. Kanamori, and T. Heaton (2009). Real-time testing of the on-site warning algorithm in southern California and its performance during the July $292008 M_{\mathrm{w}} 5.4$ Chino Hills earthquake, Geophys. Res. Lett. 36, no. 5, doi: 10.1029/ 2008 GL036366.

Böse, M., E. Hauksson, K. Solanki, H. Kanamori, Y.-M. Wu, and T. Heaton (2009). A new trigger criterion for improved real-time performance of onsite earthquake early warning in southern California, Bull. Seismol. Soc. Am. 99, 2A, 897-905.

Böse, M., T. H. Heaton, and E. Hauksson (2012). Real-time finite fault rupture detector (FinDer) for large earthquakes, Geophys. J. Int. 191, no. 2, 803-812.

Brown, H. M., R. M. Allen, M. Hellweg, O. Khainovski, D. Neuhauser, and A. Souf (2011). Development of the ElarmS methodology for earthquake early warning: Realtime application in California and offline testing in Japan, Soil Dynam. Earthq. Eng. 31, no. 2, $188-200$.

Chiou, B.-J., and R. R. Youngs (2008). An NGA model for the average horizontal component of peak ground motion and response spectra, Earthq. Spectra 24, no. 1, 173-215.

Colombelli, S., A. Zollo, G. Festa, and M. Picozzi (2014). Evidence for a difference in rupture initiation between small and large earthquakes, Nature Commun. 5, doi: 10.1038/ncomms4958.

Crowell, B. W., Y. Bock, and D. Melgar (2012). Real-time inversion of GPS data for finite fault modeling and rapid hazard assessment, Geophys. Res. Lett. 39, no. 9, 1-6, doi: 10.1029/2012GL051318.

Cua, G., and T. Heaton (2007). The Virtual Seismologist (VS) method: A Bayesian approach to earthquake early warning, in Earthquake Early Warning Systems, Springer-Verlag, Berlin, Germany, 97-132.

Cua, G. B. (2005). Creating the virtual seismologist: Developments in ground motion characterization and seismic early warning, Ph.D. Thesis, California Institute of Technology, Pasadena, California.

Ellsworth, W., and G. Beroza (1995). Seismic evidence for an earthquake nucleation phase, Science 268, no. 5212, 851-855.

Hammer, C., M. Beyreuther, and M. Ohrnberger (2012). A seismic-event spotting system for volcano fast-response systems, Bull. Seismol. Soc. Am. 102, no. 3, 948-960.

Heaton, T. H. (1985). A model for a seismic computerized alert network, Science 228, no. 4702, 987-990.

Kagan, Y. Y., and L. Knopoff (1981). Stochastic synthesis of earthquake catalogs, J. Geophys. Res. 86, no. B4, 2853-2862.

Kanamori, H. (2005). Real-time seismology and earthquake damage mitigation, Anпи. Rev. Earth Planet. Sci. 33, 195-214.

Kuyuk, H. S., and R. M. Allen (2013). Optimal seismic network density for earthquake early warning: A case study from California, Seismol. Res. Lett. 84, no. 6, 946-954.

Kuyuk, H. S., R. M. Allen, H. Brown, M. Hellweg, I. Henson, and D. Neuhauser (2013). Designing a network-based earthquake early warning algorithm for California: ElarmS-2, Bull. Seismol. Soc. Am. 84, no. 6, 946-954.

Meier, M.-A., M. Werner, J. Woessner, and S. Wiemer (2014). A search for evidence of secondary static stress triggering during the $1992 M_{\mathrm{w}} 7.3$ Landers, California, earthquake sequence, J. Geophys. Res. 119, no. 4, 3354-3370.

Minson, S., J. R. Murray, J. O. Langbein, and J. S. Gomberg (2014). Real-time inversions for finite fault slip models and rupture geometry based on high-rate GPS data, J. Geophys. Res. 119, no. 4, 3201-3231.

Odaka, T., K. Ashiya, S. Tsukada, S. Sato, K. Ohtake, and D. Nozaka (2003). A new method of quickly estimating epicentral distance and magnitude from a single seismic record, Bull. Seismol. Soc. Am. 93, no. 1, 526-532.

Olson, E. L., and R. M. Allen (2005). The deterministic nature of earthquake rupture, Nature 438, no. 7065, 212-215.

Picozzi, M., A. Zollo, P. Brondi, S. Colombelli, L. Elia, and C. Martino (2015). Exploring the feasibility of a nationwide earthquake early warning system in Italy, J. Geophys. Res. 120, no. 4, 2446-2465, doi: $10.1002 / 2014 J B 011669$

Rydelek, P., and S. Horiuchi (2006). Earth science: Is earthquake rupture deterministic? Nature 442, no. 7100, E5-E6.

Rydelek, P., C. Wu, and S. Horiuchi (2007). Comment on "Earthquake magnitude estimation from peak amplitudes of very early seismic signals on strong motion records" by Aldo Zollo, Maria Lancieri, and Stefan Nielsen, Geophys. Res. Lett. 34, no. 20, 1-3, doi: 10.1029/2007GL029387. 
Satriano, C., L. Elia, C. Martino, M. Lancieri, A. Zollo, and G. Iannaccone (2011). PRESTo, the earthquake early warning system for southern Italy: Concepts, capabilities and future perspectives, Soil Dynam. Earthq. Eng. 31, no. 2, 137-153.

Satriano, C., A. Lomax, and A. Zollo (2008). Real-time evolutionary earthquake location for seismic early warning, Bull. Seismol. Soc. Am. 98, no. 3, 1482-1494.

Simons, F. J., B. D. Dando, and R. M. Allen (2006). Automatic detection and rapid determination of earthquake magnitude by wavelet multiscale analysis of the primary arrival, Earth Planet. Sci. Lett. 250, no. 1, 214-223.

Wu, S., M. Yamada, K. Tamaribuchi, and J. Beck (2015). Multi-events earthquake early warning algorithm using a Bayesian approach, Geophys. J. Int. 200, no. 2, 789-806.

Wu, Y.-M., H. Kanamori, R. M. Allen, and E. Hauksson (2007). Determination of earthquake early warning parameters, $\tau_{c}$ and $P_{d}$, for southern California, Geophys. J. Int. 170, no. 2, 711-717.

Zhou, H.-W. (1994). Rapid three-dimensional hypocentral determination using a master station method, J. Geophys. Res. 99, no. B8, $15,439-15,455$.
Swiss Seismological Service

Institute of Geophysics

ETH Zürich

Sonneggstrasse 5

CH-8092 Zürich, Switzerland

men-andrin.meier@sed.ethz.ch

john.clinton@sed.ethz.ch

(M.-A.M., J.C.)

California Institute of Technology

Seismological Laboratory

Division of Geological and Planetary Sciences

1200 East California Boulevard

Pasadena, California 91125

heaton@caltech.edu

(T.H.)

Manuscript received 10 April 2015;

Published Online 8 September 2015 